\title{
Sentimientos de culpa y abandono de los valores paternos
}

\author{
ITZIAR ETXEBARRIA \\ Universidad del Pais Vasco

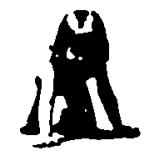 \\ Resumen
}

Este articulo trata de ser una aportación en torno a la problemática que se plantea a muchos jóvenes cuando empiezan a rechazar los valores parentales para comenzar a pensar de un modo más favorable sobre determinadas cuestiones que en su familia se consideran *moralmente incorrectas..

Se investiga la relación entre la culpa y los siguientes predictores: 1) valores morales de los progenitores, 2) tipo de disciplina parental, 3) caracteristicas del medio familiar, 4) valores de los amigos y 5) sexo de los sujetos. Además, se analizan las diferencias en el uso de las distintas técnicas disciplinarias con los varones y con las mujeres.

En la discusión se destacan los resultados relativos a las disciplinas, la culpa en la mujer y el influjo de los amigos.

Palabras clave: Culpa, Cambio de valores, Internalización moral, Disciplinas parentales, Diferencias sexuales, Pares.

\section{Guilt feelings and giving up parental values}

\section{Abstract}

The present article attempts to be a contribution to the study of problems young people face when they begin to reject parental values and to adopt a more open approach towards certain issues regarded as *morally incorrect* within their families.

The relationship between guilt and the following predictor variables was investigated: 1) parents' moral values, 2) type of parental discipline, 3) characteristics of the family environment, 4) friends' values, and s) sex of the subjects. In addition, differences in the use of discipline techniques towards males and females were analysed. In the discussion, findings related to discipline patterns, women's guilt feelings, and influence of friends are underlined.

Key words: Guilt feelings, Change in Values, Moral internalization, Parental disciplines, Sex differences, Peers.

Dirección del autor: Universidad del País Vasco. Facultad de Psicología. Apartado 1.249. San Sebastián.

Original recibido: junio 1990. Revisión recibida: noviembre 1990. Aceptado: noviembre 1990. 


\section{INTRODUCCION}

Este artículo se propone reclamar la atención y aportar alguna luz en torno a una cuestión cuyo esclarecimiento puede ser de gran interés de cara a comprender mejor algunos aspectos importantes de la evolución de la persona, y en la que, sin embargo, la Psicología de la Moral apenas si ha reparado. Se trata de la problemática que se plantea a muchos jóvenes cuando, al contacto con otras formas de pensar, de actuar, al contacto con otras ideologías, con "ciertas lecturas» y/o «malas compañías", empiezan a rechazar los valores parentales que hasta entonces - a veces sin mucha conciencia de ello- habían compartido, para comenzar a pensar de un modo diferente, más favorable, sobre determinadas cuestiones que en su familia se consideran "malas" 0 "moralmente incorrectas". Nos estamos refiriendo aquí a algo que forma parte de la experiencia de muchas personas: al fenómeno de la vivencia, en tales momentos, de ciertos sentimientos de culpa ante la puesta en práctica de los valores que racionalmente se sostienen no sólo como válidos, sino incluso a veces como mucho más correctos y adecuados que aquéllos en los que se ha sido educado (Konopka, 1983; Lo Presto, Sherman y Sherman, 1985).

Es ésta una cuestión en la que la Psicología de la Moral aún no se ha detenido mínimamente. El estudio de los procesos de cambio moral se halla de modo general (salvo en lo que respecta al juicio moral) bastante descuidado, y, desde luego, se puede decir que la Psicología de la Moral - tal como ya lo planteara Hoffman en 1980, con escaso eco- se ha olvidado casi por completo de la importancia de los tempranos condicionamientos emocionales en dichos procesos de cambio.

$\mathrm{Y}$, sin embargo, el interés del tema no debería escapársenos sobre todo si nos preocupa lo que puede ser una evolución de la persona hacia la madurez y la autonomía. Porque, si tenemos en cuenta los efectos habituales de los sentimientos de culpa, parece claro que la aparición de tales sentimientos en los momentos de cambio de valores puede suponer un freno, un obstáculo más o menos serio, para dicho cambio.

Constituye una observación común y son numerosos los trabajos tanto teóricos como empíricos -estos últimos, en los más diversos terrenosque apoyan la idea de que los sentimientos de culpa actúan como un factor de inhibición de la conducta a la que se hallan asociados (ver, especialmente, Mosher, 1979).

Pero, además, los sentimientos de culpa ejercen otro efecto que no siempre resulta tan evidente: el de generar sumisión, acatamiento, obediencia. Diversos estudios experimentales llevan a la conclusión de que la culpa incrementa la conducta de sumisión, no sólo ante la víctima, sino también ante una demanda realizada por otras personas, incluidas personas que no tienen conocimiento de que el sujeto haya cometido transgresión alguna (Brock y Becker, 1966; Wallace y Sadalla, 1966; Freedman, Wallington y Bless, 1967; Carlsmith y Gross, 1969; Yinon, Bizman, Gohen y Segev, 1976). Este sometimiento del sujeto que experimenta culpa parece producirse incluso hacia requerimientos claramente discrepantes con su propia actitud en un determinado terreno (ver a este respecto Brock y Becker, 1966).

Si esto es así, podemos pensar que el sentimiento de culpa en los momentos de cambio de valores puede actuar como un freno para dicho cam- 
bio, no sólo a través de la inhibición de las conductas congruentes con los nuevos valores, sino también motivando el plegamiento de la persona ante las demandas de los demás. La culpa que acompaña a los nuevos valores morales asumidos por el sujeto generaría en éste una disposición a complacer las demandas de los otros, aún no estando totalmente de acuerdo con su contenido, y una tendencia a plegarse a las mismas. Y una demanda está actuando en esos momentos en los sujetos desde su propio fuero interno: la demanda de los padres, la exigencia más o menos fuerte de conformidad con ellos y de sometimiento a sus normas y valores, es decir, a los antiguos valores ahora desechados por la persona. Esto explicaría el hecho de que el sujeto manifieste a veces conductas poco acordes, rezagadas, con respecto a los nuevos valores morales por los que pretende regirse. Tales conductas, aparentemente inconsistentes, incongruentes, no serían sino el reflejo del plegamiento del sujeto a dicha demanda interna.

Así pues, parece clara la relevancia que pueden tener estos sentimientos de culpa en los momentos de cambio de valores como los que aquí nos interesan. Sería, por tanto, conveniente estar atentos a su aparición. En este sentido cobra especial interés analizar qué factores pueden estar en la base de la tendencia a experimentar dichos sentimientos. ¿En qué personas tienden a darse en mayor medida?

Estas fueron las preocupaciones y éste el interrogante a los que se trató de responder a través de la investigación empírica que aquí vamos a presentar.

En esta investigación se abordaron también, como variable criterio, los sentimientos de culpa en general, no sólo en los procesos de cambio de valores. Ello, fundamentalmente, por dos razones: 1) Una, la de que es éste un aspecto en sí mismo interesante, ya que existen bastantes estudios que han tratado de analizar en general -es decir, no sólo en los sujetos que se hallan viviendo un proceso de cambio- diversos factores en relación con la culpa como «rasgo» o "disposición" de personalidad, pero muy pocos que hayan estudiado la emoción de culpa, lo que podríamos denominar culpa situacional, esto es, el sentimiento de culpa asociado a la puesta en práctica de una conducta concreta (Mosher, 1979). 2) Y otra, la de que de este modo se podría comparar lo que ocurre en el conjunto de la muestra con lo que ocurre en los sujetos que se encuentran viviendo el proceso de cambio.

Por otra parte, al reflexionar detenidamente sobre la cuestión, se pensó que tanto la culpa en general como la culpa en los procesos de cambio podían revestir características diferentes en distintas áreas de valoración moral. Por ello, ambas fueron analizadas en cuatro áreas problemáticas diferentes, todas ellas claramente relevantes: la del valor del trabajo (culpa ante "Descuido de los estudios"), la de la propiedad privada («Pequeños robos»), la religiosa («Abandono de la religión») y la sexual (culpa ante «Relaciones sexuales prematrimoniales», «Masturbación», «Relaciones sexuales circunstanciales», "Relaciones homosexuales", "Atracción homosexual», y en las mujeres, además, «Embarazo» $\mathrm{y}$ "Aborto»). 


\section{RELACION ENTRE DIVERSOS FACTORES Y LOS SENTIMIENTOS DE CULPA}

Las variables predictoras cuya relación con la culpa interesó especialmente analizar, dada su posible relevancia en los momentos de cambio de valores, fueron: 1) los valores morales de los progenitores, 2) el tipo de disciplina más frecuentemente utilizada por los progenitores con los sujetos ante diversas transgresiones de las normas familiares, 3) determinadas características del medio familiar (religiosidad y autonomía), 4) los valores de los amigos y 5) el propio sexo de los sujetos.

1. En primer lugar, parecía importante tomar en cuenta las valoraciones morales de los progenitores, en la medida en que dichas valoraciones constituyen el contenido normativo en el que fundamentalmente ha sido socializado el sujeto. Extrañamente, apenas hay trabajos empiricos que hayan analizado la relación entre este factor y los sentimientos de culpa, pero diversos planteamientos - como los de Wright (1974), Hoffman (1980), o el mismo Freud y el psicoanálisis- sugieren cierta correlación negativa entre ambas variables: al margen de los valores sustentados por el sujeto, cuanto más negativas (más desfavorables) sean las valoraciones de los padres sobre determinadas cuestiones, mayores serán los sentimientos de culpa de los sujetos en relación a dichas cuestiones.

2. La literatura empirica acerca del influjo de las diversas prácticas disciplinarias sobre la internalización moral y los sentimientos de culpa es muy extensa y bastante compleja. Aquí tan sólo destacaremos los aspectos más relevantes de la misma en relación con la presente investigación.

En los estudios realizados durante las décadas de los años 50 y 60 se utilizaron distintas clasificaciones de las técnicas disciplinarias parentales. Sin embargo, a partir del trabajo de Hoffman y Saltzstein (1967) la mayor parte de las investigaciones distinguen tres tipos de disciplina: la afirmación de poder, la retirada de amor y la inducción. Dentro de este último tipo de disciplina en algunos trabajos se distinguen dos variantes: la inducción referida a los pares y la inducción referida a los progenitores ${ }^{1}$.

Pues bien, podemos decir que, en conjunto, los estudios que han analizado el influjo de estas técnicas disciplinarias sobre la internalización moral llevan a la conclusión de que las orientaciones morales caracterizadas por la independencia del sujeto con respecto a las sanciones externas y fuertes sentimientos de culpa se hallan asociadas con el uso frecuente, por parte de la madre, de técnicas de disciplina inductiva -especialmente con inducciones que resaltan las consecuencias dolorosas que la conducta del hijo comporta en otras personas - y con la expresión frecuente de afecto por parte de aquélla en situaciones no disciplinarias (Hoffman y Saltzstein, 1967; Zahn-Waxler, Radke-Yarrow y King, 1979; Hoffman, 1963, 1970, 1977, 1980, 1982, 1983; Eisikovits y Sagi, 1982).

De las investigaciones realizadas se desprende que son las prácticas inductivas de la madre, y no tanto las del padre, las que ejercen efectos diferenciales en la internalización moral y en la tendencia a experimentar culpa de los hijos (para una discusión sobre este punto, véase Hoffman, 1970, 1980, 1983).

3. Autores de diversas disciplinas - filósofos, sociólogos, psicólogos- 
han señalado numerosas veces cómo la religión cristiana se basa en buena medida en la inducción de temor al castigo y sentimientos de culpa para conseguir una fuerte internalización de la moral religiosa. Este tipo de planteamientos ha llevado a algunos autores a suponer que debe de darse una relación muy estrecha entre religiosidad y predisposición a experimentar fuertes sentimientos de culpa ante la transgresión.

Aunque las bases para sustentar tal suposición parecen suficientemente firmes y el estudio de esta relación podría ser de gran interés, es muy poco lo que se ha investigado para responder a esta cuestión. No obstante, los estudios existentes (Peretti, 1969; Joyce, 1977; Schmidt, 1988) apoyan la idea de que la religiosidad se halla relacionada con la tendencia a sentir culpa.

4. Es también poco lo que se ha teorizado, y menos aún lo que se ha investigado empíricamente, sobre el influjo de los pares en la evolución moral del adolescente y el joven. No obstante, la investigación disponible sugiere que, cuando los valores de los pares y de los padres no coinciden, es más probable que la interacción con los iguales tenga un efecto debilitador, en lugar de fortalecedor, de las inhibiciones de carácter moral que éste había adquirido en el marco familiar (Sherif, Harvey, White, Hood y Sherif, 1961; Bandura, Grusec y Menlove, 1967; Devereaux, 1970; revisiones de Hoffman, 1970, 1980; Finn y Doyle, 1983; Sack, Keller y Hinkle, 1984; Chen y Shi, 1987).

5. En cuanto a posibles diferencias sexuales en culpa, se ha llegado, con amplio apoyo empírico, a la conclusión de que las mujeres adolescentes y adultas poseen una orientación moral más internalizada y una mayor tendencia a experimentar sentimientos de culpa que los varones (Peretti, 1969; Biaggio, 1969; Heying, Korabik y Munz, 1975; Breen y Prociuk, 1976; Hoffman, 1975, 1977, 1980, 1983; Evans, 1984; Bovbjerg, 1985; Lalos, Lalos, Jacobsson y Von Schoultz, 1986; Klass, 1988; Perry, Perry y Weiss, 1989). No obstante, algunos autores -como Heying y col., 1975circunscriben esta tendencia a ciertas conductas específicas, concretamente, a las de índole sexual. Si estas conclusiones pueden generalizarse o no a otras culturas más allá de la occidental es una cuestión que está todavía por dilucidar y exige un mayor ahondamiento a partir del estudio de la misma en culturas no occidentales.

En cualquier caso, ¿cómo puede explicarse esta mayor tendencia a los sentimientos de culpa en las mujeres, al menos en nuestra cultura? Hemos visto en el punto 2 que dichos sentimientos de culpa se hallan asociados con el uso frecuente, por parte de la madre, de técnicas de disciplina inductiva y con la expresión frecuente de afecto por parte de aquélla en situaciones no disciplinarias. Pues bien, en las muestras del estudio de Hoffman arriba citado (1975) las madres eran más afectuosas con las hijas que con los hijos y usaban con ellas más técnicas inductivas y menos técnicas de afirmación de poder que con los hijos. En otro estudio, Zussman (1975, 1978) halló igualmente que las mujeres, en general, se veían sometidas a menos prácticas de afirmación de poder y a más prácticas inductivas que los varones. Por otra parte, hay datos adicionales que sugieren que las prácticas inductivas podrían ser, además, más efectivas en las mujeres que en los varones: se ha constatado que aquéllas, en la temprana edad, son más em- 
páticas (Feshback y Feshback, 1969; Hoffman y Levine, 1976), y la efectividad de este tipo de técnicas se basa precisamente en gran medida en su capacidad de activación de respuestas empáticas (Hoffman, 1963; Hoffman y Saltzstein, 1967). Dada la mayor proclividad hacia la empatía en las mujeres, las inducciones despertarán en ellas mayores respuestas empáticas y serán por tanto más efectivas.

Teniendo en cuenta todo lo que acabamos de ver sobre el estado de la investigación acerca de cada predictor, el presente estudio se planteó, en síntesis, la siguiente hipótesis. Los sentimientos de culpa ante las diversas problemáticas concretas ("Masturbación", "Descuido de los estudios", "Pequeños robos"...), tanto en el conjunto de la muestra como, dentro de ella, en los sujetos que manifestaran cambio, serían:

1. Más intensos cuanto más negativas (desfavorables) fueran las valoraciones morales de sus progenitores sobre dichas cuestiones.

2. Más intesos cuantas más fueran las prácticas de «inducción referida a los progenitores" y menos fueran las prácticas de «razonamiento" a las que se hubieran visto sometidos por parte de sus progenitores (en el apartado siguiente se explicará el significado de esta última categoría).

Además, los sentimientos de culpa de los sujetos mostrarían una relación, más estrecha con las disciplinas maternas que con las paternas.

3. Más intensos cuanto mayor fuera el grado de "moralidad-religiosidad" y menor el de «autonomía" de su ambiente familiar.

4. Más intensos también en las mujeres que en los varones.

5. En cambio, dichos sentimientos de culpa serían menos intensos cuanto más positivas (favorables) fueran las valoraciones morales de los amigos.

Además de esto, en el trabajo se trató de explorar si en los sentimientos de culpa de los sujetos pesaban más las disciplinas utilizadas por los progenitores en la infancia o las utilizadas en la adolescencia.

Asimismo, intentando aportar algo de cara a explicar las diferencias sexuales observables en este campo, se analizaron las diferencias en el uso de las distintas técnicas disciplinarias con los varones y con las mujeres: en este sentido se hipotetizaba que éstas se verían sometidas a más inducciones que los varones.

\section{METODO}

\section{Sujetos}

\section{Descripción de la muestra}

Tras la realización de un estudio piloto para detectar un grupo de edad en el que hubiera bastantes sujetos que revelaran estar experimentando en ese preciso "momento" ( «desde hace menos de 2 años») un proceso de cambio como el que interesaba, la muestra estuvo constituida por 252 estudiantes de BUP, COU, $3 .^{\circ}$ y $4 .^{\circ}$ de FP de ambos sexos: 133 varones y $119 \mathrm{mu}-$ jeres, de 16 a 19 años (media de edad, 17 años). 
Definición del grupo de cambio

Tras reflexionar y ver que no se contaba con una medida adecuada de la variable «momento de cambio», el grupo de cambio en cada problemática específica («Rel. sex. prematrimoniales», "Masturbación», etc.), quedó definido de un modo más amplio, sin tener en cuenta dicho aspecto, como el grupo constituido por los sujetos que sostenían valoraciones morales positivas sobre dicha problemática con progenitores que mantenían valoraciones negativas sobre la misma.

Los valores de los sujetos se midieron a través de 14 ítems que hacían referencia, en conjunto, a las cuatro áreas problemáticas de interés en el estudio. Al sujeto se le pedía que opinara sobre cada una de las cuestiones. Las alternativas de respuesta eran cuatro: "Muy mal" (MM), "Un poco mal» (M), "Algo bien" (B) y "Muy bien» (MB). Por ejemplo, entre otros ítems, se pedía al sujeto su opinión sobre: «Las relaciones sexuales entre personas del mismo sexo», «Los pequeños robos en grandes almacenes, establecimientos, lugares de trabajo, instituciones oficiales...», etc.

Los valores de los progenitores, tras intentar recabar la colaboración de los padres y encontrar enormes dificultades para ello, se midieron volviendo a plantear posteriormente al sujeto las mismas cuestiones y preguntándole esta vez en cada una de ellas por la opinión de sus padres al respecto.

A partir de las respuestas de los sujetos a estos dos conjuntos de ítems, el grupo de cambio en cada problemática específica quedó constituido por los sujetos que valoraban positivamente la problemática en cuestión ( $B$ o $M B$ ), con progenitores que, según su propia información, la valoraban negativamente $(M \circ M M)$ : Progenitores - Hijos +.

\section{Procedimiento}

La evaluación de cada sujeto en las diversas variables se realizó a través de un cuestionario. Dicho cuestionario constaba básicamente de cuatro partes: culpa, valores (sujetos, padres y amigos), disciplinas, y ambiente familiar. En esta última parte se utilizó la Escala de Clima Social en la Familia (FES) de R. H. Moos (1974/1984). Las demás medidas fueron elaboradas ex profeso para la presente investigación.

Ya hemos visto cómo se midieron los valores de los sujetos y los de los progenitores. Del mismo modo se midieron los de los amigos.

Los sentimientos de culpa en relación con las diversas problemáticas — «Descuido de los estudios», «Pequeños robos», etc.- se midieron, tal como es usual en este tipo de estudios (ver, por ej., Hoffman, 1975, 1977, 1980), a través de una serie de pequeñas historias semiproyectivas -10 en total, tantas como problemáticas - en las cuales se presentaba a los sujetos una situación en la que se cometía lo que, para los sujetos del grupo de cambio, constituiría una transgresión o una falta desde el punto de vista de los valores desechados, pero no desde el punto de los valores actuales. Se promovía la identificación del sujeto con el/la protagonista de dichas historias, se le preguntaba cómo se sentiría en dicha situación, y se le pedía que, si creía que sentiría algún grado de sentimiento de culpa, puntuara su intensidad en una escala de siete puntos.

Así, por ejemplo, la historia en la que se trataban de medir los sentimientos de culpa en relación a una conducta de robo planteaba lo siguiente: 
*Un chico anda mal de dinero; con lo que normalmente maneja tiene que privarse de muchas cosas que le gustan. Un día decide probar suerte $y$, aunque un poco nervioso, roba algunas cosas en unos grandes almacenes.»

A continuación se le decía al sujeto (en este caso varón):

„Imagínate que tú eres ese chico y que acabas de robar, ¿cómo te sentirías?»

«Si crees que sentirías algún grado de sentimiento de culpa, puntúa su intensidad en la siguiente escalaw:

$\begin{array}{lllllllllll}\text { Ningún s. de culpa } & 1 & 2 & 3 & 4 & 5 & 6 & 7 & \text { Mucho s. de culpa }\end{array}$

Las diversas historias abarcaban en conjunto las distintas problemáticas de interés en el trabajo. Cada una de ellas reflejaba la puesta en práctica de una de las conductas sobre la que los sujetos, en la parte del cuestionario referente a valores - siempre posteriormente, para controlar que esta segunda medición no contaminara la de la culpa-, daban su opinión moral.

Para medir la disciplina parental —en la línea de los trabajos de Hoffman al respecto- se planteaban al sujeto diversas situaciones disciplinarias de la vida real (cuatro típicas de la adolescencia y otras tres de la infancia), se favorecía la identificación del sujeto con el/la protagonista que desencadenaba dichas situaciones, y se le pedía que señalara en dos listas separadas, la $1 .^{2}$, la $2 .^{2}$ y la $3 .^{2}$ conductas más frecuentes de su padre y de su ma$d r e$ en ese tipo de situaciones.

Una de las situaciones (en este caso de la adolescencia), por ejemplo, planteaba lo siguiente:

«Un chico lleva una temporada que no asiste a la Iglesia. Sus padres se enteran de que no va a Misa y un día surge el tema en casa.»

Inmediatamente se planteaba:

"Imagínate que tú eres ese chico. ¿Cuáles son la 1.2, la 2." y la 3." conductas más frecuentes de tu padre y de tu madre cuando se plantea esta situación en tu casa?»

Cada una de las listas — una referente al padre, y otra a la madre- que seguían a esta pregunta contenía, presentadas al azar, 12 conductas parentales diferentes, 3 por cada una de las categorías contempladas en el estudio: «afirmación de poder», "retirada de amor", «inducción referida a los progenitores» $y$ «razonamiento».

Como puede observarse, se añadió una categoría nueva a las habituales en este tipo de estudios: el razonamiento. La razón de ello fue que la sospecha de que las anteriores categorías no agotaban el conjunto de los diversos tipos de disciplinas parentales posibles se vio confirmada en el estudio piloto antes mencionado, cuando muchos sujetos señalaron que los comportamientos de sus progenitores en dichas situaciones disciplinarias no se asemejaban a ninguna de las conductas de la lista propuesta -que en dicho estudio piloto incluía sólo las otras tres categorias-, sino que se trataban de prácticas claramente basadas en el diálogo y la explicación de por qué la conducta estaba mal. Como veremos en los resultados, la distinción de esta categoría se revelaría después claramente pertinente.

Por último, hay que señalar que tanto las situaciones disciplinarias de la adolescencia como las de la infancia hacían referencia en su contenido «transgresor» a las diversas problemáticas de interés en el estudio. Así, los 
encuentros disciplinarios de la infancia se desencadenaban por problemas de "Actividad sexual», «Malas notas» y "Mala contestación", y los de la adolescencia por "Relaciones sexuales», «Mal curso», «No ir a misa», e «Insulto a los padres». No se incluyó ninguna situación disciplinaria relacionada con conductas de «Pequeños robos» por considerarse que los encuentros disciplinarios motivados por este tipo de temática no son algo demasiado común. En su lugar, como se puede observar, se incluyeron, tanto en la infancia como en la adolescencia, además de las situaciones más estrechamente relacionadas con las problemáticas de interés central en el estudio, situaciones relativas a ofensas verbales de los hijos a los padres.

\section{RESULTADOS}

Básicamente, primero se analizó la relación de cada uno de los predictores con los sentimientos de culpa por separado, y posteriormente se hicieron una serie de análisis globales a fin de ver hasta qué punto el conjunto de las variables consideradas explicaba la varianza que se da en los sentimientos de culpa y - lo que más interesaba en estos análisis globales-el peso relativo de cada una de ellas.Tanto estos últimos analisis como los primeros se realizaron, por un lado, sobre el conjunto de la muestra, y, por otro, sobre los sujetos de los grupos de cambio en cada problemática (salvo el primer análisis a nivel bivariado, en que esto último, como veremos, no era posible).

A continuación trataremos de exponer de forma sintética, lo más claramente posible, los resultados de este conjunto de análisis. Dado que la presentación de todas las tablas de resultados exigiría mucho espacio, nos limitaremos a presentar, en los casos en que los análisis se efectuaron por un lado con el conjunto de la muestra y, por otro, con los grupos de cambio, las tablas de los análisis efectuados con los sujetos en proceso de cambio, por ser éstos el objeto de interés prioritario de este trabajo. En los análisis globales finales, presentaremos también una tabla-resumen de los resultados obtenidos en el conjunto de la muestra ${ }^{2}$.

\section{Valoraciones morales de los progenitores}

En el conjunto de la muestra, las correlaciones halladas (correlaciones simples y parciales) sugieren en algunas problemáticas - todas ellas de carácter sexual- una cierta relación, aunque débil, entre los valores de los progenitores y la culpa de los sujetos, al margen de los valores que estos últimos tengan sobre dichas cuestiones: cuanto más desfavorables son las valoraciones de sus padres al respecto, mayores son los sentimientos de culpa del sujeto.

El análisis del influjo de esta variable sobre los sentimientos de culpa de los sujetos de los grupos de cambio no se realizó ya que no era posible, al ser éstos, por su propia definición, muy homogéneos en dicha variable (los sujetos de estos grupos, como ya se ha dicho, tenían progenitores que sólo opinaban $\mathrm{M}$ o $\mathrm{MM}$, es decir, desfavorablemente, sobre las diversas cuestiones).

En este caso, por tanto, presentamos los resultados obtenidos en el conjunto de la muestra (ver Tabla I). 
TABLA I

Valoraciones morales de los progenitores y sentimientos de culpa. Correlaciones

\begin{tabular}{|c|c|c|c|c|c|c|c|c|c|c|}
\hline Items de culpa & $(\mathrm{N})$ & $\begin{array}{l}\text { Cor. simp. } \\
\text { v. prog. }\end{array}$ & $\begin{array}{l}\text { Cor. parc. } \\
\text { v. prog. }\end{array}$ & $\mathbf{T}$ & Sign. & $\begin{array}{l}\text { Cor. simp. } \\
\text { v. suj. }\end{array}$ & $\begin{array}{l}\text { Cor. parc. } \\
\text { v. suj. }\end{array}$ & $\mathbf{T}$ & Sign. & $\begin{array}{l}\text { Correlación } \\
\text { v. p. - v. s. }\end{array}$ \\
\hline $\begin{array}{l}\text { C. rel. prem. } \\
\text { C. masturbac. } \\
\text { C. rel circ. } \\
\text { C. rel. hom. } \\
\text { C. atrac. hom. } \\
\text { C. embarazo } \\
\text { C. aborto } \\
\text { C. desc. estud. } \\
\text { C. aban. relig. } \\
\text { C. robo }\end{array}$ & $\begin{array}{l}(247) \\
(237) \\
(243) \\
(231) \\
(237) \\
(118) \\
(118) \\
(247) \\
(237) \\
(249)\end{array}$ & $\begin{array}{l}-0,15 \\
-0,32 \\
-0,31 \\
-0,11 \\
-0,05 \\
-0,26 \\
-0,25 \\
-0,11 \\
0,12 \\
-0,04\end{array}$ & $\begin{array}{r}-0,04 \\
-0,11 \\
-0,14 \\
-0,03 \\
0,04 \\
-0,16 \\
-0,12 \\
-0,09 \\
0,01 \\
0,06\end{array}$ & $\begin{array}{r}-0,65 \\
-1,95 \\
-2,48 \\
-0,53 \\
0,73 \\
-1,84 \\
-1,40 \\
-1,38 \\
0,11 \\
1,04\end{array}$ & $\begin{array}{l}0,516 \\
0,052(t) \\
0,014^{*} \\
0,595 \\
0,469 \\
0,068(t) \\
0,164 \\
0,170 \\
0,914 \\
0,299\end{array}$ & $\begin{array}{l}-0,38 \\
-0,45 \\
-0,51 \\
-0,25 \\
-0,26 \\
-0,29 \\
-0,35 \\
-0,19 \\
0,30 \\
-0,38\end{array}$ & $\begin{array}{r}-0,35 \\
-0,33 \\
-0,42 \\
-0,22 \\
-0,26 \\
-0,20 \\
-0,27 \\
-0,18 \\
0,28 \\
-0,38\end{array}$ & $\begin{array}{l}-5,89 \\
-5,76 \\
-7,70 \\
-3,44 \\
-4,08 \\
-2,34 \\
-3,17 \\
-2,84 \\
4,48 \\
-6,51\end{array}$ & $\begin{array}{l}0,0000 \\
0,0000 \\
0,0000 \\
0,0007 \\
0,0001 \\
0,0213 \\
0,0019 \\
0,0049 \\
0,0000 \\
0,0000\end{array}$ & $\begin{array}{l}0,31 * * \\
0,49 * * \\
0,37 * * \\
0,36 * * \\
0,36 * * \\
0,37 * * \\
0,37 * * \\
0,12 \\
0,34 * * \\
0,27 * *\end{array}$ \\
\hline
\end{tabular}

V. prog.: valoraciones morales de los progenitores sobre cada una de las problemáticas.

V. suj.: valoraciones de los skjetos.

\section{Disciplina parental}

Tanto en los grupos de cambio como en el conjunto de la muestra, los sentimientos de culpa resultan más fuertes cuantas más son las «inducciones referidas a los progenitores" y menos las prácticas de «razonamiento" a las que se ha visto sometido el sujeto. En cambio, y tal como también se había hipotetizado, apenas se revelan diferencias en los sentimientos de culpa en relación con el uso por parte de los progenitores de técnicas de «afirmación de poder» y "retirada de amor» (Análisis Discriminantes, ver Tabla II, para los grupos de cambio; para facilitar su lectura, en esta tabla, al igual que en las Tablas $V$ y VI relativas a las disciplinas, se incluyen solamente aquellas variables de disciplina en las que los estadísticos fueron significativos o tendenciales).

Por otra parte, ni en los grupos de cambio ni en la muestra se observa un mayor peso general de las disciplinas maternas frente a las paternas. Tampoco aparecen claras diferencias entre las disciplinas de la adolescencia $y$ las de la infancia.

\section{Caracteristicas del ambiente familiar}

En la muestra, los resultados de las pruebas $t$ de Student revelan que, en la mayoría de las problemáticas, a mayor «moralidad-religiosidad", más culpa, mientras que a mayor «autonomía», menos culpa.

En los grupos de cambio, la relación positiva entre «moralidad-religiosidad" y culpa sólo se observa en dos problemáticas (Tabla III).

\section{Diferencias sexuales}

Tanto en los sujetos en proceso de cambio como en el conjunto de la muestra (Tabla IV, para los grupos de cambio), las mujeres experimentan, efectivamente, sentimientos de culpa más intensos que los varones (incluso cuando se controla, a través de un análisis de covarianza como el realizado, la variable valores), pero sólo en el terreno de las conductas sexuales.

Esta conclusión presenta una clara consistencia con los resultados obtenidos en relación: 1) a los efectos de las distintas prácticas disciplinarias utilizadas por los progenitores y 2) al diferente uso que de dichas prácticas hacen éstos cuando se dirigen a los varones y cuando lo hacen a las mujeres. 


\section{TABLA II}

Disciplina parenteral y sentimientos de culpa en los grupos de cambio. Análisis discriminante

\begin{tabular}{|c|c|c|c|c|c|}
\hline Items de culpa & Disciplinas & C. baja & C. alta & $\mathbf{F}$ & Sign. \\
\hline C. rel. prem. & $\begin{array}{l}\text { In. rel. sex. madre } \\
\text { In. rel. sex. padre }\end{array}$ & $\begin{array}{l}1,71 \\
0,93\end{array}$ & $\begin{array}{l}2,73 \\
1,73\end{array}$ & $\begin{array}{l}3,527 \\
3,438\end{array}$ & $\begin{array}{l}0,0647 \\
0,0680\end{array}$ \\
\hline C. masturbac. & $\begin{array}{l}\text { Rz. rel. sex. madre } \\
\text { In. act. sex. madre } \\
\text { Rz. rel. sex. padre } \\
\text { Rz. act. sex. madre }\end{array}$ & $\begin{array}{l}3,46 \\
1,39 \\
4,00 \\
2,96\end{array}$ & $\begin{array}{l}1,83 \\
2,75 \\
2,58 \\
1,67 .\end{array}$ & $\begin{array}{l}5,353 \\
4,001 \\
3,662 \\
3,641\end{array}$ & $\begin{array}{l}0,0262 \\
0,0526 \\
0,0632 \\
0,0640\end{array}$ \\
\hline C. rel. circ. & $\begin{array}{l}\text { Rz. rel. sex. padre } \\
\text { In. rel. sex. padre } \\
\text { Af.-pod. act. sex. madre } \\
\text { Af. pod. rel. sex. padre } \\
\text { In. act. sex. madre } \\
\text { Rz. rel. sex. madre }\end{array}$ & $\begin{array}{l}4,67 \\
0,58 \\
1,12 \\
0,05 \\
1,21 \\
3,72\end{array}$ & $\begin{array}{l}2,97 \\
1,41 \\
0,34 \\
0,44 \\
2,06 \\
2,87\end{array}$ & $\begin{array}{r}21,600 \\
8,123 \\
5,271 \\
5,147 \\
4,756 \\
3,151\end{array}$ & $\begin{array}{l}0,0000 \\
0,0057 \\
0,0246 \\
0,0262 \\
0,0324 \\
0,0800\end{array}$ \\
\hline C. rel. hom. & $\begin{array}{l}\text { In. act. sex. padre } \\
\text { Rz. rel. sex. madre }\end{array}$ & $\begin{array}{l}0,60 \\
3,30\end{array}$ & $\begin{array}{l}1,62 \\
2,19\end{array}$ & $\begin{array}{l}9,798 \\
3,850\end{array}$ & $\begin{array}{l}0,0029 \\
0,0554\end{array}$ \\
\hline C. atrac. hom. & In. act. sex. padre & 0,67 & 1,50 & 3,535 & 0,0682 \\
\hline C. embarazo & $\begin{array}{l}\text { In. rel. sex. madre } \\
\text { Rz. act. sex. madre }\end{array}$ & $\begin{array}{l}2,21 \\
2,50\end{array}$ & $\begin{array}{l}3,50 \\
0,50\end{array}$ & $\begin{array}{l}3,923 \\
3,733\end{array}$ & $\begin{array}{l}0,0631 \\
0,0692\end{array}$ \\
\hline C. aborto & & & & - & - \\
\hline C. desc. estuid. & $\begin{array}{l}\text { Af. pod. mal. not. madre } \\
\text { Ret. am. mal. not. padre }\end{array}$ & $\begin{array}{l}1,25 \\
0,25\end{array}$ & $\begin{array}{l}0,33 \\
0,00\end{array}$ & $\begin{array}{l}3,907 \\
3,481\end{array}$ & $\begin{array}{l}0,0570 \\
0,0716\end{array}$ \\
\hline C. aban. relig. & & & & - & - \\
\hline
\end{tabular}

In.: inducción. Rz.: razonamiento. Af. pod.: afirmación de poder. Ret. am.: retirada de amor. Rel. sex.: situación disciplinaria relativa a un problema de erelaciones sexuales» (situación de la adolescencia). Act. sex.: situación relativa a un problema de "actividad sexual. (infancia). Mal. not.: situación relativa a un problema de "malas notas" (infancia). Asi, "In. rel. sex. madre*: inducción utilizada por las madres en la situación disciplinaria relativa a erelaciones sexuales.

1. Como ya hemos visto, los sentimientos de culpa elevados muestran relación con el uso frecuente de prácticas "inductivas» y el uso infrecuente de prácticas de "razonamiento" por parte de los progenitores.

2. Pues bien, en el estudio se reveló que en general, es decir, por término medio en las diversas situaciones disciplinarias, en la adolescencia las mujeres se ven sometidas por parte de ambos progenitores a más prácticas de carácter inductivo que los varones, mientras que éstos reciben -al menos por parte del padre — más prácticas de razonamiento que las mujeres (Prueba t de Student, Tabla V).

Además, curiosamente, estas diferencias en el uso de las diversas prácticas disciplinarias con los hijos y con las hijas fueron particularmente claras en un tipo de encuentros disciplinarios: los relativos a las "Relaciones sexuales" en la adolescencia. En ellos ambos progenitores utilizan más inducciones y menos razonamiento con las mujeres que con los varones (Prueba t de Student, Tabla VI). 


\section{TABLA III}

Ambiente familiar y sentimientos de culpa en los sujetos de los grupos de cambio. Prueba st" de Student

\begin{tabular}{|c|c|c|c|c|c|}
\hline Items de culpa & Clima Fam. & C. baja & C. alta & $\mathbf{T}$ & Sign. \\
\hline C. rel. prem. & $\begin{array}{l}\text { Mor-relig. } \\
\text { Autonomía }\end{array}$ & $\begin{array}{l}(64) \\
3,64 \\
4,87\end{array}$ & $\begin{array}{c}(13) \\
4,08 \\
4,09\end{array}$ & $\begin{array}{l}0,92 \\
1,28\end{array}$ & $\begin{array}{l}0,362 \\
0,205\end{array}$ \\
\hline C. masturbac. & $\begin{array}{l}\text { Mor-relig. } \\
\text { Autonomía }\end{array}$ & $\begin{array}{l}(32) \\
3,50 \\
4,66\end{array}$ & $\begin{array}{l}(16) \\
3,81 \\
4,00\end{array}$ & $\begin{array}{l}0,56 \\
1,06\end{array}$ & $\begin{array}{l}0,578 \\
0,294\end{array}$ \\
\hline C. rel. circ. & $\begin{array}{l}\text { Mor-relig. } \\
\text { Autonomía }\end{array}$ & $\begin{array}{l}(47) \\
3,42 \\
4,89\end{array}$ & $\begin{array}{c}(40) \\
3,47 \\
4,47\end{array}$ & $\begin{array}{l}0,41 \\
1,02\end{array}$ & $\begin{array}{l}0,896 \\
0,310\end{array}$ \\
\hline C. rel. hom. & $\begin{array}{l}\text { Mor-relig. } \\
\text { Autonomia }\end{array}$ & $\begin{array}{l}(37) \\
3,57 \\
4,70\end{array}$ & $\begin{array}{c}(26) \\
3,73 \\
3,85\end{array}$ & $\begin{array}{l}0,36 \\
1,59\end{array}$ & $\begin{array}{l}0,721 \\
0,116\end{array}$ \\
\hline C. atrac. hom. & $\begin{array}{l}\text { Mor-relig. } \\
\text { Autonomía }\end{array}$ & $\begin{array}{c}(25) \\
3,32 \\
4,64\end{array}$ & $\begin{array}{c}(25) \\
3,72 \\
4,20\end{array}$ & $\begin{array}{l}0,80 \\
0,75\end{array}$ & $\begin{array}{l}0,395 \\
0,456\end{array}$ \\
\hline C. aborto & $\begin{array}{l}\text { Mor-relig. } \\
\text { Autonomía }\end{array}$ & $\begin{array}{c}(18) \\
2,89 \\
3,72\end{array}$ & $\begin{array}{l}(15) \\
3,80 \\
4,80\end{array}$ & $\begin{array}{l}1,64 \\
1,70\end{array}$ & $\begin{array}{l}0,111 \\
0,099(t)\end{array}$ \\
\hline C. desc. estud. & $\begin{array}{l}\text { Mor-relig. } \\
\text { Autonomia }\end{array}$ & $\begin{array}{l}(15) \\
3,20 \\
5,67\end{array}$ & $\begin{array}{l}(24) \\
3,42 \\
4,33\end{array}$ & $\begin{array}{l}0,44 \\
2,43\end{array}$ & $\begin{array}{l}0,661 \\
0,020 *\end{array}$ \\
\hline C. aban. relig. & $\begin{array}{l}\text { Mor-relig. } \\
\text { Autonomia }\end{array}$ & $\begin{array}{c}(33) \\
2,69 \\
5,12\end{array}$ & $\begin{array}{c}(12) \\
4,42 \\
4,58\end{array}$ & $\begin{array}{l}3,84 \\
0,75\end{array}$ & $\begin{array}{l}0,000 * \\
0,457\end{array}$ \\
\hline C. robo & $\begin{array}{l}\text { Mor-relig. } \\
\text { Autonomía }\end{array}$ & $\begin{array}{c}(9) \\
7,22 \\
10,22\end{array}$ & $\begin{array}{r}(8) \\
11,00 \\
7,63\end{array}$ & $\begin{array}{c}Z \\
-1,60 \\
-1,09\end{array}$ & $\begin{array}{l}0,110 \\
0,276\end{array}$ \\
\hline C. embarazo & $\begin{array}{l}\text { Mor-relig. } \\
\text { Autonomía }\end{array}$ & $\begin{array}{c}(17) \\
11,24 \\
13,62\end{array}$ & $\begin{array}{c}(8) \\
17,78 \\
13,28\end{array}$ & $\begin{array}{c}Z \\
-2,14 \\
-0,11\end{array}$ & $\begin{array}{l}0,033 * \\
0,913\end{array}$ \\
\hline
\end{tabular}

\section{Valoraciones morales de los amigos}

Tanto en los grupos de cambio como en el conjunto de la muestra (Correlaciones simples y parciales, Tabla VII, análisis en los grupos de cambio), los valores de los amigos revelan una correlación moderada, negativa, con los sentimientos de culpa de los sujetos ante varias cuestiones: cuanto más positivas son las valoraciones morales de sus amigos en torno a dichas cuestiones, menores son los sentimientos de culpa del sujeto.

Además, en este factor se apunta un fenómeno curioso: en algunas problemáticas (en «Rel. homosexuales», «Descuido estudios» $y$ «Pequeños ro- 


\section{TABLA IV}

Diferencias sexuales en culpa en los sujetos del grupo de cambio de cada temática. Análisis de covarianza

\begin{tabular}{lcccccccc}
\hline & \multicolumn{9}{c}{$\begin{array}{c}\text { X ajustadas } \\
\text { Items de culpa }\end{array}$} & X var. & X muj. & var. & muj. & F & Sign. & \multicolumn{1}{c}{ F cov. Sign. cov. } \\
\hline C. rel. prem. & $1,74(54)$ & $2,51(67)$ & 1,71 & 2,54 & 9,25 & $0,003 *$ & 9,32 & 0,003 \\
C. masturbac. & $2,81(52)$ & $3,72(58)$ & 2,72 & 3,81 & 10,19 & $0,002 *$ & 10,94 & 0,001 \\
C. rel. circ. & $2,48(82)$ & $4,09(57)$ & 2,56 & 4,00 & 21,32 & $0,000 *$ & 22,38 & 0,000 \\
C. rel. hom. & $2,71(52)$ & $3,59(58)$ & 2,69 & 3,61 & 5,99 & $0,016 *$ & 0,28 & 0,598 \\
C. atrac. hom. & $3,15(52)$ & $4,00(58)$ & 3,11 & 4,04 & 6,44 & $0,013 *$ & 1,35 & 0,248 \\
C. desc. estud. & $5,35(49)$ & $5,29(38)$ & 5,34 & 5,29 & 0,02 & 0,895 & 0,67 & 0,416 \\
C. aban. relig. & $2,53(34)$ & $2,48(33)$ & 2,54 & 2,47 & 0,02 & 0,880 & 4,69 & 0,034 \\
C. robo & $3,08(13)$ & $4,00(14)$ & 3,01 & 4,07 & 1,65 & 0,212 & 0,34 & 0,562 \\
\hline
\end{tabular}

\section{TABLA V}

Diferencias entre el tipo de disciplinas más frecuentemente utilizadas en general con los varones y con las mujeres. Prueba *t* de Student

\begin{tabular}{lllcccl}
\hline & \multicolumn{2}{l}{ Disciplinas } & X var. & X muj. & T & \multicolumn{1}{c}{ Sign. } \\
\hline Infancia & \multirow{2}{*}{ Padre } & Af. poder & 1,19 & 0,97 & 1,96 & $0,0505^{*}$ \\
& & Ret. amor & 0,44 & 0,58 & 1,86 & $0,0634(\mathrm{t})$ \\
Adolesc. & Padre & Inducción & 0,79 & 1,01 & 3,34 & $0,0009^{*}$ \\
& & Razonam. & 3,45 & 3,11 & 2,81 & $0,0051^{*}$ \\
& Madre & Inducción & 1,37 & 1,58 & 2,26 & $0,0242^{*}$ \\
\hline
\end{tabular}

\section{TABLA VI}

Diferencias entre el tipo de disciplinas más frecuentemente utilizadas en las diversas situaciones disciplinarias con los varones y con las mujeres. Prueba «t»

\begin{tabular}{|c|c|c|c|c|c|c|}
\hline \multicolumn{3}{|c|}{ Disciplinas infancia } & \multirow{2}{*}{$\begin{array}{c}X \text { var. } \\
0,39 \\
3,63\end{array}$} & \multirow{2}{*}{$\begin{array}{c}\text { X muj. } \\
0,77 \\
3,23\end{array}$} & \multirow{2}{*}{$\begin{array}{c}T \\
3,38 \\
1,84\end{array}$} & \multirow{2}{*}{$\begin{array}{l}\text { Sign. } \\
0,0008 * \\
0,0670(t)\end{array}$} \\
\hline Act. sex. & Padre & $\begin{array}{l}\text { Ret. amor } \\
\text { Razonam. }\end{array}$ & & & & \\
\hline Mal. not. & Madre & Af. poder & 0,88 & 0,61 & 2,29 & $0,219 *$ \\
\hline Mal. cont. & Padre & Inducción & 0,95 & 1,30 & 2,53 & $0,0116 *$ \\
\hline \multicolumn{3}{|c|}{ Disciplinas adolescencia } & $X$ var. & $\mathrm{X}$ muj. & $\mathbf{T}$ & Sign. \\
\hline Rel. sex. & Madre & $\begin{array}{l}\text { Af. poder } \\
\text { Inducción } \\
\text { Ret. amor } \\
\text { Razonam. } \\
\text { Af. poder } \\
\text { Inducción } \\
\text { Razonam. }\end{array}$ & $\begin{array}{l}0,20 \\
0,69 \\
0,64 \\
4,16 \\
0,16 \\
1,12 \\
3,85\end{array}$ & $\begin{array}{l}0,56 \\
1,21 \\
1,04 \\
3,03 \\
0,33 \\
1,93 \\
3,04\end{array}$ & $\begin{array}{l}3,63 \\
4,23 \\
2,96 \\
5,74 \\
2,31 \\
5,18 \\
4,25\end{array}$ & $\begin{array}{l}0,0003 * \\
0,0000 * \\
0,0032 * \\
0,0000 * \\
0,0213 * \\
0,0000 * \\
0,0000 *\end{array}$ \\
\hline Mal curso & & & & & - & - \\
\hline Insulto & $\begin{array}{l}\text { Padre } \\
\text { Madre }\end{array}$ & $\begin{array}{l}\text { Inducción } \\
\text { Ret. amor }\end{array}$ & $\begin{array}{l}0,70 \\
0,76\end{array}$ & $\begin{array}{l}0,94 \\
1,06\end{array}$ & $\begin{array}{l}2,04 \\
2,32\end{array}$ & $\begin{array}{l}0,0421 * \\
0,0206 *\end{array}$ \\
\hline No ir Misa & Madre & Af. poder & 0,13 & 0,05 & 1,88 & $0,0607(t)$ \\
\hline
\end{tabular}




\section{TABLA VII}

Valores morales de los amigos y sentimientos de culpa en los sujetos en proceso de cambio. Correlaciones

\begin{tabular}{|c|c|c|c|c|c|c|c|c|c|c|}
\hline Items de culpa & $(\mathbf{N})$ & $\begin{array}{l}\text { Cor. simp. } \\
\text { v. amig. }\end{array}$ & $\begin{array}{l}\text { Cor. parc. } \\
\text { v. amig. }\end{array}$ & $\mathbf{T}$ & Sign. & $\begin{array}{l}\text { Cor. simp. } \\
\text { v. suj. }\end{array}$ & $\begin{array}{l}\text { Cor. parc. } \\
\text { v. suj. }\end{array}$ & $\mathbf{T}$ & Sign. & $\begin{array}{l}\text { Correlación } \\
\text { v. a. - v. s. }\end{array}$ \\
\hline $\begin{array}{l}\text { C. rel. prem. } \\
\text { C. masturbac. } \\
\text { C. rel circ. } \\
\text { C. rel. hom. } \\
\text { C. atrac. hom. } \\
\text { C. embarazo } \\
\text { C. aborto } \\
\text { C. desc. estud. } \\
\text { C. aban. relig. } \\
\text { C. robo }\end{array}$ & $\begin{array}{l}(120) \\
(110) \\
(137) \\
(108) \\
(109) \\
(52) \\
(53) \\
(83) \\
(65) \\
(26)\end{array}$ & $\begin{array}{r}-0,18 \\
-0,08 \\
-0,19 \\
-0,19 \\
-0,09 \\
-0,07 \\
0,01 \\
-0,17 \\
-0,05 \\
-0,46\end{array}$ & $\begin{array}{r}-0,16 \\
0,03 \\
-0,14 \\
-0,18 \\
-0,07 \\
-0,04 \\
0,03 \\
-0,18 \\
-0,07 \\
-0,45\end{array}$ & $\begin{array}{r}-1,84 \\
0,30 \\
-1,72 \\
-1,91 \\
-0,76 \\
-0,29 \\
0,24 \\
-1,61 \\
-0,59 \\
-2,49\end{array}$ & $\begin{array}{l}0,069(t) \\
0,763 \\
0,087(t) \\
0,059(t) \\
0,448 \\
0,774 \\
0,814 \\
0,111 \\
0,557 \\
0,020 *\end{array}$ & $\begin{array}{r}-0,26 \\
-0,29 \\
-0,35 \\
-0,06 \\
-0,12 \\
-0,22 \\
-0,14 \\
-0,07 \\
0,27 \\
0,11\end{array}$ & $\begin{array}{r}-0,24 \\
-0,28 \\
-0,33 \\
-0,03 \\
-0,10 \\
-0,21 \\
-0,14 \\
-0,09 \\
0,28 \\
-0,05\end{array}$ & $\begin{array}{r}-2,80 \\
-3,04 \\
-4,09 \\
-0,35 \\
-1,10 \\
-1,53 \\
-1,01 \\
-0,87 \\
2,28 \\
-0,29\end{array}$ & $\begin{array}{l}0,006 * \\
0,003 * \\
0,000 * \\
0,727 \\
0,274 \\
0,132 \\
0,318 \\
0,388 \\
0,026 * \\
0,772\end{array}$ & $\begin{array}{l}0,09 \\
0,38 * * \\
0,16 \\
0,18 \\
0,18 \\
0,11 \\
0,11 \\
-0,14 \\
0,09 \\
-0,36\end{array}$ \\
\hline
\end{tabular}

V. amig.: valoraciones morales de los amigos sobre cada una de las problemáticas.

V. suj.: valoraciones de los sujetos.

bos» en los grupos de cambio, y en «Rel. homosexuales» en la muestra general), la culpa de los sujetos parece guardar una relación más estrecha con la valoración moral que de ellas tienen los amigos que con la del propio sujeto.

Análisis global de la relación entre el conjunto de variables consideradas y los sentimientos de culpa

A partir de los análisis globales (análisis discriminantes y análisis de regresión múltiple; aquí sólo incluiremos las tablas-resumen de los discriminantes), podemos extraer las siguientes conclusiones:

\section{En el conjunto de la muestra (Tabla VIII):}

- Las variables predictoras en algunas problemáticas - «Rel. sex. prematrimoniales», «Masturbación», «Rel. sex. circunstanciales», «Embarazo» y "Aborto"- explican una proporción considerable de la varianza que se da en los sentimientos de culpa, mientras que en otras explican una proporción más pobre.

- Las variables que en general tienden a presentar un mayor peso en el conjunto de ítems de culpa son: además de las valoraciones morales de los propios sujetos, el sexo, la moralidad-religiosidad y la autonomia.

\section{En los grupos de cambio (Tabla IX):}

- Aquí, los resultados no son significativos en todas las problemáticas. En los ítems en que lo son - "Rel. sex. prematrimoniales", "Rel. sex. circunstanciales", "Embarazo" y "Masturbación", tendencial-, las variables consideradas revelan una buena capacidad explicativa, en algunos casos bastante alta.

Estos resultados cobran un mayor interés si se tiene en cuenta que en los análisis con los grupos de cambio no entraron como variables predictoras ni los valores de los progenitores ni, los que aún es más importante, los de los propios sujetos, debido a que en tales variables los sujetos de los grupos de cambio, por su propia definición, eran muy homogéneos.

La falta de significatividad de la función discriminante y de la ecuación de regresión múltiple en el resto de las problemáticas de culpa podría ex- 


\section{TABLA VIII}

Relación entre el conjunto de variables y la culpa en la muestra. Análisis discriminante

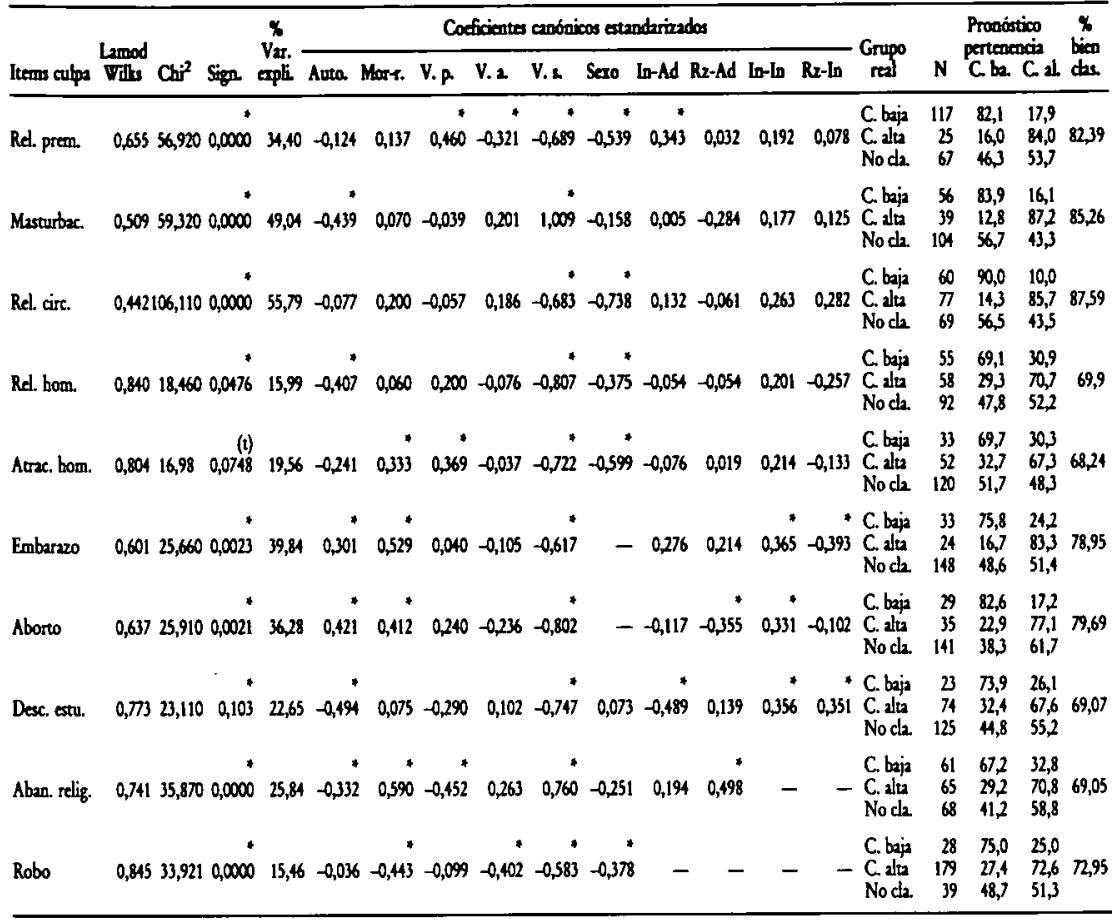

Auto.: Autonomia. Mor-r: Moralidad-religiosidad. V. p.: valoraciones morales de los progrenitores. V. a.: Valoraciones de los amigos. V. s.: valoraciones de los sujetos. In-Ad.: inducciones utilizadas por los progenitores en la adolescencia. Rz-Ad.: razonamiento utilizado en la adolescencia. In-In: inducción en la infancia. Rz-In: Razonamiento en la infancia.

plicarse tanto por el hecho de que las variables predictoras que quedaron en estos análisis no serían capaces de explicar por sí solas - sobre todo, sin tener en cuenta los valores de los sujetos- la varianza de la culpa ante dichas problemáticas en los sujetos en proceso de cambio, como por el hecho de que en los grupos de cambio se contara con pocos sujetos en tales problemáticas. La primera explicación parece la más plausible, ya que - como se ha visto - en otras problemáticas, con un número similar de sujetos en los grupos de cambio, los estadísticos sí resultaron significativos.

- En los grupos de cambio las variables con más peso en general son: nuevamente, sexo y «moralidad-religiosidad»; además, en el análisis discriminante destacaron las disciplinas parentales (*inducción" $y$ "razonamientow) y, en la regresión, los valores de los amigos.

Por lo tanto, en respuesta a la cuestión que especialmente se trataba de responder con este trabajo, podemos decir que los sentimientos de culpa en los procesos de cambio ante las problemáticas mencionadas serán más intensos:

- En las mujeres.

- En los sujetos cuyo ambiente familiar se caracterice por un alto grado de "moralidad-religiosidad». 


\section{TABLA IX}

Relación entre el conjunto de variables y la culpa en los grupos de cambio. Análisis discriminante

\begin{tabular}{|c|c|c|c|c|c|c|c|c|c|c|c|c|c|c|c|c|c|}
\hline \multirow[b]{2}{*}{ Items cupa } & \multirow{2}{*}{$\begin{array}{l}\text { Lamod } \\
\text { Wilks }\end{array}$} & \multirow[b]{2}{*}{$\mathrm{Ch}^{2}$} & \multirow[b]{2}{*}{ Sign. } & \multirow[b]{2}{*}{$\%$} & \multicolumn{8}{|c|}{ Cocficientes canónicos entandarizados } & \multirow{2}{*}{ Grupo } & \multirow[b]{2}{*}{$\mathbf{N}$} & \multicolumn{2}{|c|}{ Prooósticos } & \multirow{2}{*}{ bien } \\
\hline & & & & & Aura. & More. & V. 2. & Sexo & In-Ad & Rz-Ad & In-ln & $R_{2}-\ln$ & & & c. & C. al. & \\
\hline Rel. prem. & 0,577 & 34,538 & 0,0000 & 42,20 & $-0,382$ & 0,175 & $-0,692$ & $-0,522$ & 0,617 & 0,336 & $-0,033$ & $-0,047$ & $\begin{array}{l}\text { C. baja } \\
\text { C. alu } \\
\text { No ch }\end{array}$ & $\begin{array}{l}58 \\
11 \\
40\end{array}$ & $\begin{array}{r}86,2 \\
9,1 \\
47,5\end{array}$ & $\begin{array}{l}13,8 \\
90,8 \\
52,5\end{array}$ & $86, \%$ \\
\hline Masturbac. & 0,663 & 14,363 & $\begin{array}{r}(t) \\
0,0728\end{array}$ & 33,66 & $-0,263$ & 0,408 & $-0,334$ & $-0,416$ & $-0,529$ & $-0,899$ & 0,737 & 0,365 & $\begin{array}{l}\text { C. baja } \\
\text { C. alu } \\
\text { No cha }\end{array}$ & $\begin{array}{l}28 \\
13 \\
56\end{array}$ & $\begin{array}{l}78,6 \\
23,1 \\
60,7\end{array}$ & $\begin{array}{l}21,4 \\
76,9 \\
39,3\end{array}$ & 78,05 \\
\hline Rel. circ. & 0,594 & 35,851 & 0,0000 & 40,52 & 0,104 & 0,47 & 0,098 & $-0,867$ & 0,162 & $\begin{array}{r}* 0,300 \\
\end{array}$ & 0,482 & 0,594 & $\begin{array}{l}\text { C. baja } \\
\text { C. ala } \\
\text { No cla. }\end{array}$ & $\begin{array}{l}43 \\
32 \\
47\end{array}$ & $\begin{array}{l}81,4 \\
25,0 \\
46,8\end{array}$ & $\begin{array}{l}18,6 \\
75,0 \\
53,2\end{array}$ & 78,67 \\
\hline Rel. hom. & 0,794 & 10,098 & 0,2582 & 20,51 & $-0,106$ & 0,086 & $-0,284$ & $-0,593$ & $-0,368$ & $-0,240$ & 0,698 & 0,153 & $\begin{array}{l}\text { C. baja } \\
\text { C. alka } \\
\text { No da. }\end{array}$ & $\begin{array}{l}29 \\
21 \\
43\end{array}$ & $\begin{array}{l}65,5 \\
38,1 \\
32,6\end{array}$ & $\begin{array}{l}34,5 \\
51,9 \\
57,4\end{array}$ & 54,00 \\
\hline Atrac. hom. & 0,748 & 8,961 & 0,345 & 25,10 & 0,163 & 0,336 & $-0,154$ & $-0,119$ & $-0,273$ & 0,819 & 0,437 & 0,185 & $\begin{array}{l}\text { C. baja } \\
\text { C. ala } \\
\text { No da. }\end{array}$ & $\begin{array}{l}17 \\
20 \\
56\end{array}$ & $\begin{array}{l}82,4 \\
35,0 \\
42,9\end{array}$ & $\begin{array}{l}17,6 \\
65,0 \\
57,1\end{array}$ & 7,97 \\
\hline Embarzzo & 0,391 & 14,541 & 0,0424 & 50,86 & & 1,229 & $-0,039$ & - & 1,056 & 0,571 & 0,464 & $-0,043$ & $\begin{array}{l}\text { C. baja } \\
\text { C. alta } \\
\text { No cla. }\end{array}$ & $\begin{array}{r}15 \\
6 \\
59\end{array}$ & $\begin{array}{r}93,3 \\
0,0 \\
54,2\end{array}$ & $\begin{array}{r}6,7 \\
10,0 \\
45,3\end{array}$ & 95,24 \\
\hline Aborto & 0,818 & 4,701 & 0,6464 & 18,13 & 0,709 & 0,755 & $-0,273$ & - & $-0,104$ & $-0,24$ & 0,257 & 0,188 & $\begin{array}{l}\text { C. baja } \\
\text { C. ata } \\
\text { No da. }\end{array}$ & $\begin{array}{l}18 \\
11 \\
51\end{array}$ & $\begin{array}{l}72,2 \\
45,5 \\
43,1\end{array}$ & $\begin{array}{l}27,8 \\
\mathbf{5 4 , 5} \\
\mathbf{5 6 , 9}\end{array}$ & 65,52 \\
\hline Desc. estud. & 0,776 & 5,310 & 0,612 & 22,31 & $-0,827$ & 0,121 & $-0,164$ & 0,117 & $-0,369$ & 0,458 & 0,151 & 0,255 & $\begin{array}{l}\text { C. baja } \\
\text { C. alta } \\
\text { No da. }\end{array}$ & $\begin{array}{l}12 \\
19 \\
45\end{array}$ & $\begin{array}{l}75,0 \\
21,1 \\
46,7\end{array}$ & $\begin{array}{l}25,0 \\
78,9 \\
53,3\end{array}$ & 77,42 \\
\hline Aban. relig. & 0,769 & 7,615 & 0,2676 & 23,10 & $-0,188$ & 0,952 & $-0,123$ & 0,49 & 0,128 & 0,179 & - & & $\begin{array}{l}\text { C. baja } \\
\text { C. alu } \\
\text { No da. }\end{array}$ & $\begin{array}{l}24 \\
10 \\
19\end{array}$ & $\begin{array}{l}66,7 \\
30,0 \\
57,9\end{array}$ & $\begin{array}{l}33,3 \\
70,0 \\
42,1\end{array}$ & 67,65 \\
\hline Robo & 0,526 & 6,093 & 0,1923 & 37,42 & 0,500 & 1,000 & $-0,311$ & $-0,821$ & - & - & - & & $\begin{array}{l}\text { C. baja } \\
\text { C. ala } \\
\text { No cla. }\end{array}$ & $\begin{array}{l}9 \\
8 \\
8\end{array}$ & $\begin{array}{l}66,7 \\
12,5 \\
37,9\end{array}$ & $\begin{array}{l}33,3 \\
87,5 \\
12,5\end{array}$ & 76,47 \\
\hline
\end{tabular}

Auto: Antonomia. Mor-r.: moralidad-religiosidad. V. a.: valoraciones morales de los amigos. Ind-Ad: inducciones utilizadas por los progenizores en la adolexcencia. Rz-Ad: razonamiento witizado en la adolescencia. In-In: inducción en la infancia. Rz-In: razonamiento en la infancsa.

- En los sujetos que, en los encuentros disciplinarios con sus progenitores, se hayan visto sometidos a más «inducciones referidas a los progenitores* y a menos prácticas de "razonamiento».

En cambio, los sentimientos de culpa de esos sujetos serán menores cuanto más favorable sea la valoración moral de sus amigos sobre dichas cuestiones.

\section{COMENTARIO}

En conjunto, como se puede observar, los resultados sobre la relación entre cada predictor y la culpa se presentan en la línea de trabajos anteriores que han abordado esta misma relación, pero a un nivel general - no específicamente con muestras de sujetos en proceso de cambio-, y con culpa "rasgo" - no «situacional»- como variable criterio.

La única conclusión de la literatura empírica que no se confirma es la relativa al mayor peso de las disciplinas maternas frente a las paternas. No obstante, esto podría deberse al modo en que en el estudio se midieron dichas disciplinas, que probablemente favorecía el mimetismo del sujeto en sus respuestas sobre unas y otras. 
Por otra parte, es interesante señalar que en este trabajo -como en el de Heying y col., 1975 - las diferencias en culpa entre varones y mujeres, tanto en el conjunto de la muestra como específicamente entre los sujetos de los grupos de cambio, sólo emergieron en el terreno de las conductas sexuales.

Otra observación general a realizar es que, básicamente, parecen ser los mismos los factores que más pesan sobre la culpa en general y sobre la culpa en los procesos de cambio: el sexo y la «moralidad-religiosidad».

Pero pasemos a comentar, siquiera brevemente, algunos resultados concretos. De los resultados obtenidos podríamos destacar como más interesantes, por un lado, los relativos a las disciplinas, y por otro, en relación más directa con la cuestión que especialmente interesaba en el trabajo, esto es, con la problemática de la culpa en los procesos de cambio de valores, los relativos a la mujer y al influjo de los amigos. Vayamos por partes.

1. La distinción propuesta en este trabajo entre «inducción» y «razonamiento" parece encontrar claro sentido, tanto teórico como práctico. Como hemos visto, uno y otro tipo de prácticas actúan sobre la culpa en sentido justamente contrario. Por otra parte, difieren también en sus implicaciones educativas: así, si no se quiere obstaculizar el camino de la autonomía con una culpa racionalmente injustificada, una culpa que podriamos denominar «a-racional», habría que desistir del uso «chantajista» de técnicas inductivas referidas a los padres para dar cada vez mayor cabida, también en los encuentros disciplinarios, a la práctica del diálogo.

También por lo que respecta a las disciplinas, merecen especial atención los resultados relativos al diferente uso de unas y otras prácticas disciplinarias con los hijos y con las hijas (resultados que, como se habrá podido observar, presentan una clara consistencia con los de otros estudios anteriores: Hoffman, 1975; Zussman, 1975, 1978), y muy en particular los relativos al modo en que estas prácticas difieren con uno y otro sexo especialmente en el terreno de la conducta sexual.

2. Parece claro que la experiencia de culpa, al menos en ciertas problemáticas (las de índole sexual), y en nuestra cultura, tiende a presentar una mayor intensidad en las mujeres.

$\mathrm{Si}$, junto a los resultados obtenidos en este trabajo sobre los efectos de los diversos tipos de disciplinas y el uso diferencial de éstas con uno y otro sexo, tomamos en cuenta los datos de la literatura empírica - mencionados anteriormente- que sugieren una mayor efectividad de las prácticas inductivas en las mujeres, si además tenemos en cuenta que, probablemente, éstas se ven sometidas a encuentros disciplinarios relativos a su conducta sexual con mayor frecuencia que los varones, el fenómeno no habrá ya de extrañarnos. Parece haber base suficiente para sostener que dichos sentimientos de culpa más intensos en la mujer responden, al menos en gran parte, a unas prácticas educativas bien determinadas.

Por otra parte, y retomando lo que planteábamos en la introducción respecto a los efectos de los sentimientos de culpa, no se nos debería escapar el hecho de que tales sentimientos más intensos en la mujer pueden suponer para ésta dificultades añadidas a las que ya de por sí plantean a la persona los procesos de cambio de valores. Puede que aquí radique en buena medida la explicación de la experiencia, común a tantas mujeres, de la es- 
pecial dificultad que este tipo de procesos de cambio parecen comportar para ellas.

3. Por lo que se refiere a los amigos, vemos que éstos pueden favorecer el avance del joven en el terreno de la autonomía moral, al aliviarle del peso de la culpa ante conductas que éste personalmente considera moralmente correctas. En este sentido, su influjo podría considerarse muy positivo.

Pero la consideración que este efecto nos merezca dependerá de nuestra opinión particular sobre los valores hacia los que los amigos ayuden a avanzar al joven; así, del mismo modo, el grupo de amigos podría aliviar los sentimientos de culpa de aquél ante conductas claramente rechazables desde todo punto de vista. Por otra parte, tal consideración dependerá también de que dicha ayuda sea realmente eso, una ayuda para dar el salto a la plena autonomía moral, y no el comienzo de una nueva dependencia con respecto a otro código externo, simplemente diferente.

En la investigación que aquí se ha presentado sólo se analizó, más allá del marco familiar, la influencia de los amigos, pero la interpretación de este resultado parece llevarnos a pensar que, probablemente, de un modo más general, el mismo efecto podrían ejercer otras personas o entidades que sostengan los mismos valores que el sujeto acaba de abrazar (incluidos, por ej., dentro del mismo marco familiar, hermanos mayores que hayan experimentado el cambio antes que él).

Quizás sea esto lo que explique el que el tipo de cambios a los que aquí nos hemos referido actualmente resulten menos traumáticos que hace tan sólo una década. Hoy en día amplios sectores sociales muestran opiniones mucho más favorables respecto a ciertas conductas, como las que aquí se han considerado, que hasta ahora hace bien poco una gran mayoría consideraba «inmorales».

Sin embargo, esta problemática sigue teniendo plena actualidad para muchos jóvenes. Esperemos haber contribuido con este trabajo a un cierto esclarecimiento y una mejor comprensión de la misma.

\section{Notas}

1 Afirmación de poder: En esta categoría se engloban aquellas prácticas que implican el uso de la fuerza física, la retirada de ciertos premios o privilegios al niño, o la amenaza de cualquiera de estas dos cosas.

Retirada de amor: Incluye todas aquellas técnicas en las que los progenitores expresan su desaprobación o enfado por la mala conducta del niño de un modo directo pero no físico, ignorando a éste, rechazándole, negándose a hablarle o escucharle, diciéndole que ya no le quieren, etc.

Inducción: Incluye fundamentalmente prácticas que tratan de dirigir la atención del niño hacia el daño que su conducta ha provocado en algún otro. $\mathrm{E}_{j} .:$ « $\mathrm{i}$ No te das cuentas de que lo que le has hecho le ha dejado muy triste?" (inducción referida a los pares), "Me vas a matar a disgustos (inducción referida a los progenitores).

2 Los lectores interesados en una información y una discusión más pormenorizadas de los análisis realizados y los datos resultantes, pueden solicitarlo a la autora. 


\section{Referencias}

BandurA, A.; Grusec, J. E. y Menlove, F. L. (1967). «Some determinants of self-monitoring reinforcement systems». Joumal of Personality and Social Psychology, S, 449-445.

BIAGGIO, A. M. B. (1969). EInternalized versus externalized guilt: a cross-cultural study*. Joumal of Social Psychology, 78, 147-149.

BOVBJERG, A. (1985). «Women's guilt x. Udkast, 13, 182-205.

BREEN, L. J. y PROCIUK, T. J. (1976). *Internal-external locus of control and guilt». Journal of Clinical Psychology, 32, 301-302.

BROCK, T. C. y BECKER, L. A. (1966). „Debriefing» and susceptibility to subsequent experimental manipulations. Journal of Experimental Social Psychology, 2, 314-323.

CARLSMITH, J. M. y Gross, A. E. (1969). *Some effects of guilt on compliance*. Journal of Personality and Social Psychology, 2, 232-239.

CHEN, X. y SHI, R. (1987). "An experimental study of two factors affecting students' moral behavior». Acta Psychologica Sinica, 19, 57-62.

DeverEauX, E. C. (1970). . The role of peer-group experience in moral development». En J. P. Hill (ed.), Minnesota Symposia on Child Psichology (vol. 4). Minneapolis: University of Minnesota Press.

EICKE-SPENGLER, M. (1988). "Shame and guilt feelings in women». Zeitschrift fur Psychoanalytische Theorie und Praxis, 3, 77-93.

EISIKOVITS, Z. y SAGI, A. (1982). *Moral development and discipline encounter in delinquent and nondelinquent adolescents*. Joumal of Youth and Adolescence, 11, 217-230.

Evans, R. G. (1984). «Hostility and sex guilt: Perceptions of self and others as a function of gender and sex-role orientation*, Sex Roles, 10, 207-215.

FESHBACK, N. D. y FESHBACK, S. (1969). "The relationship between empathy and agression in two age groups*. Developmental Psychology, 1, 102-107.

FINN, E. J. y DOYLE, R. E. (1983). *The effect of three experimental conditions on the moral judgements of 9th-grade students». Counseling and Values, 27, 150-159.

FREEDMAN, J. L., WALLINGTON, S. A. y BLESS, E. (1967). «Compliance without pressure: the effect of guiltw. Journal of Personality and Social Psychology, 7, 117-124.

FreUd, S. (1973). Obras completas, Madrid: Biblioteca Nueva.

Helkama, K. (1983). *The development of moral reasoning and moral values». Acta Psychologica Fennica, 9, 99-111.

HeYING, R. H., KoRABIK, K. y MUNZ, D. C. (1975). «Sex differences in expected guilt reactions to hypothetical behaviors of sexual, hostile, and moral substance». Perceptual and Moral Skills, 40, 409-410.

HOFFMAN, M. L. (1963). «Childrearing practices and moral development: Generalizations from empirical research. Chil Development, 34, 295-318.

- (1970). *Moral Developmentw. En P. H. Mussen (ed.), Carmichael's Handbook of Child Psychology (vol. 2). Nueva York: Wiley.

- (1975). *Sex differences in moral internalization and values*. Journal of Personality and Social Psychology, 32, 720-729.

- (1977). «Moral internalization: Current theory and research». En L. Berkowitz (ed.), Advances in Experimental Social Psychology (vol. 10). Nueva York: Academic Press.

- (1980). «Moral development in adolescence». En J. Adelson (ed.), Handbook of adolescent psychology. Nueva York: Wiley.

- (1982). «Development of prosocial motivation: Empathy and guiltm. En Eisenberg-Berg (ed.), The development of prosocial behavior. Nueva York: Academic Press.

- (1983). «Desarrollo moral y conducta». Infancia y aprendizaje. Monografia, 3, 13-36.

HofFMAN, M. L. y LEVINE, L. E. (1976). "Early sex differences in empathy». Developmental Psychology, 12, 557-558.

HoffMan, M. L. y Saltzstein, H. D. (1967). «Parent discipline and the child's moral developmentm. Journal of Personality and Social Psychology, 5, 45-57.

JOYCE, J. P. (1977). *Differences between guilt proneness and anxiety proneness on field independence, locus of control, empathy and religiosity». Dissertation Abstracts Intemational, 1884-B. (Orden No. 77-22, 341).

KLASS, E. T. (1988). *Cognitive behavioral perspectives on women and guilt. Special Issue: Cognitive-behavior therapy with women*. Journal of Rational Emotive and Cognitive Behavior Therapy, 6, 23-32.

KONOPKA, G. (1983). «Adolescent suicide». Excepcional cbildren, 49, 390-394.

Lalos, A.; LAlos, O.; JaCOBSSON, L. y VON SCHOUlTZ, B. (1986). «Depression, guilt and isolation among infertile women and their partners». Joumal of Psychosomatic Obstretics and Gynaecology, S, 197-206.

Lo Presto, Ch. T., Sherman, M. F. y Sherman, N. C. (1985). «The effects of a masturbation seminar on high school males' attitudes, false beliefs, guilt and behavior*. Journal of Sex Research, 21, 142-156.

Moos, R. H. (1984). «Clima social: Familia». En R. H. Moos, B. S. Moos y E. J. Trickett 
(ed.), Escalas de clima social: Familia, trabajo, instituciones penitenciarias, centro escolar. Madrid: TEA. (Publicación original, 1974).

MOSHER, D. L. (1979). «The meaning and measurement of guilt». En C. E. Izard (ed.), Emotions in personality and psychopatbology. Nueva York: Plenum Publishing Corporation.

PARISH, Th. S. y NUNN, G. D. (1988). "The importance of the family in forming life values and personal values*. Journal of Psychology, 122, 519-121.

PerETTI, P. O. (1969). «Guilt in moral development: A comparative study*. Psychological Reports, 25, 739-745.

Perry, D. G., Perry, L. C. y WeISS, R. J. (1989). «Sex differences in the consequences that children anticipate for aggression». Development Psychology, 25, 312-319.

SACK, A. R., KELLER, J. F. y HINKLE, D. E. (1984). «Premarital sexual intercourse: A test of the effects of peer group, religiosity and sexual guiltw. Jourmal of Sex Research, 20,168-185.

SAPOZHNIKOVA, L. S. (1985). «Development of moral values in adolescents». Voprosy Psikbologii, 1, 50-56.

SCHMIDT, P. F. (1988). «Moral values of adolescents: Public versus Christian schools». Journal of Psychology and Christianity, 7, 50-54.

SHERIF, M.; HARVEY, O. J.; WHITE, B. J.; HOOD, W. R. y SHERIF, C. (1961). Intergroup conflict and cooperation: The robber's cave experiment. Norman, Oklahoma: University Book Exchange.

WALLACE, J. y SADALLA, E. (1966). «Behavioral consequences of transgression: I. The effects of social recognition*. Journal of Experimental Research in Personality, 1, 187-194.

WRIGHT, D. (1974). Psicologia de la conducta moral. Barcelona: Planeta.

YINON, Y.; BIZMAN, A.; GOHEN, S. y SEGEV, A. (1976). «Effects of guilt-arousal communications on volunteering to the civil guard: A field experiment". Bulletin of the Psychonomic Society, 7, 493-494.

ZAHN-WAXLER, C.; RADKE-YARROW, M. y KING, R. M. (1979). «Childrearing and children's prosocial initiations toward victims of distressw. Child Development, 50, 319-330.

ZUSSMAN, J. U. (1975). Demographic factors influencing parental discipline tecbniques. Trabajo presentado a la reunión de la American Psychological Association, Chicago.

- (1978). *Relationship of demographic factors to parental discipline techniques». Developmental Psichology, 14, 685-686.

\section{Extended Summary}

The present investigation aims to study the problems young people face when they begin to reject parental values and to adopt a more open approach towards certain issues regarded as "morally incorrect" within their families. It concerns the phenomenon of guilt feelings experienced when certain values, rationally regarded as valid, are put into practice. This guilt may check or be and obstacle to such processes of change. It is therefore of interest to question and analyse which factors underlie the tendency to experiment such feelings. An empirical study is presented with a view to answering this issue.

Guilt feelings in general, and not only guilt in processes of change, was the study's criterion variable. Both of these were analysed in relation to four problem areas: importance of work, private property, religion, and sexual behaviour.

The relationship between guilt and the following predictor variables was investigated: 1) parents' moral values, 2) type of parental discipline, 3 ) characteristics of the family environment (religiosity and autonomy), 4) friends' values, and 5) sex of the subjects.

The following hypothesis was tested: Guilt feelings resulting from various problems ("Masturbation», «Petty thefts», etc.), both in the sample as a whole and in individual subjects manifesting change will: 
1) Increase in intensity with increasing negative (unfavourable) parental moral opinions about these issues.

2) Increase in intensity with more parental "Induction" and less "Reasoning". (The latter is a new category proposed in this study with a view to adding it to the usual "Induction", «Love withdrawal», and "Power assertion" categories.)

3) Increase in intensity with an increasing degree of Moral-Religious behaviour and a decreasing degree of Autonomy in the family environment.

4) Also be more intense in female than in male subjects.

5) decrease in intensity with increased positive (favourable) moral opinions from friends.

The study also attempted to explore if either childhood or adolescence parental disciplines weigh more on the subjects' guilt feelings. In addition, differences in the use of various disciplines on males and females were analysed with a view to explain observed sexual differences in this field. It is hypothesized that females will be subjected to more induction than males.

The sample were 252 students ( 133 males and 119 females, between 16-19 yrs.) The «changing group» in each specific problem («Petty thieving", "Masturbation», etc.), was defined as the group of subjects who had positive moral opinions on a particular issue while their parents' views were negative.

Subjects were assessed with the use of a questionnaire convering the four following areas: guilt; subjects', parents', and friends' values; types of discipline; and family environment. The latter was evaluated with Moos' (1974-84) Family Environment Scale (FES). All other measures were specifically designed for the present study.

First, the relationship of each predictor variable with subjects' guilt feelings was analysed separately. Subsequently, a series of global analyses were carried out in order to find out to what extent the set of variables selected in the study accounted for the variance found in subjects' guilt feelings, and - what was of even more interest - the relative weight of each variable.

In summary, global analyses carried out produced the following results: 1) In the sample as a whole, sex, Morality-Religiosity, and Autonomy were the variables which in general tended to carry more weight on the group of items of subjects' guilt feelings, together with the moral opinions of the subjects themselves. 2) In the «changing groups» results were not significant for all problems studied. Variables for items found to be significant revealed an explanatory ability which was in some cases quite high (even though neither parents' values nor, what is more important, subjects' values were entered as variables). Once again, variables carrying more weight in the "changing groups" are sex and Morality-Religiosity. Furthermore, results from discriminative analysis and multiple regression highlighted the importance of parental disciplines and friends' values respectively.

Overall, results on the relationship between each predictor variable and subjects' guilt feelings are in accordance with previous work studying the same relation but at a more general level. That is, they do not specifically deal with samples of subjects undergoing changes. The usual criterion variable in other works has been «dispositional" guilt and not «situational» guilt as in the present investigation. 
Finally, in the discussion the results considered of particular interest are: 1) those related to discipline measures, specifically, those concerning "Reasoning» techniques - a category proposed in the present work and firmly supported by the results. 2) those bearing a closer relationship with the aims of this work, that is, guilt feelings in processes of change: guilt in women, and the influence of friend's opinions on the subjects' guilt. 